Johannes G. Schmidta

Severin Bühlmann ${ }^{b}$

Ying Shao ${ }^{c}$

Akademische Vertreter der Toxikologie haben die wichtige Aufgabe, toxische Substanzen $\mathrm{zu}$ identifizieren. Klinische Erfahrungen und klinische Differenzierungen fehlen ihnen aber weitgehend, sodass sie in ihr Spezialgebiet hineinwachsen mit dem Denken, dass Gift immer Gift und ein Karzinogen immer karzinogen sei. Doch sogar Wasser ist toxisch, wenn es beim ungeeigneten Patienten im ungeeigneten Kontext verwendet wird (Wasserintoxikation). In der Chinesischen Medizin spielt die wirtsbezogene Effektmodifikation eine entscheidende Rolle. Das heisst, dass die Konstitution des Patienten mitentscheidet, wie ein Mittel wirkt. Schmeiser und Cornelius scheinen hingegen ganz im Denkschema von schädlichen und unschädlichen Substanzen verhaftet $\mathrm{zu}$ sein, sodass sie

${ }^{a}$ Stiftung Paracelsus heute und

Praxiszentrum Meinradsberg, Einsiedeln,

bPhytax-Labor, Schlieren,

cPraxiszentrum Meinradsberg, Einsiedeln, Schweiz

\title{
Erwiderung zum Kommentar von PD Dr. Heinz H. Schmeiser und Dr. Michael G. Cornelius: Schweiz Z Ganzheitsmed 2013;25: DOI: 10.1159/000346563
}

auch den epidemiologischen Begriff der Effektmodifikation missverstehen. Das zeigt den akademischen Reduktionismus, der wissenschaftlich unzulässige Generalisierungen hervorbringt.

Die erwähnte taiwanesische Studie zeigt im Übrigen Folgendes: Ein Drittel aller Taiwanesen schluckt Kräuter, die Aristolochiasäure enthalten. Doch nur sehr wenige Taiwanesen entwickeln letztlich ein Urothelkarzinom, wenn auch häufiger als in anderen Ländern. Und nur bei 55\% dieser Karzinome zeigen sich die typischen Veränderungen, die man auf Aristolochiasäure zurückführt. Wir sind einverstanden, dass AristolochiaPflanzen nicht bedenkenlos eingesetzt werden können. Im modernen Taiwan werden nicht traditionelle Aristolochia-Arten verwendet, die man in China verboten hat. Auch die Beliebtheit von Rezepturen wie Long- dan Xie Gan Tang ist bedenklich, denn isolierte Leberhitze ist selten. Und die völlig falsche Verwendung von Aristolochia in den belgischen Schlankheitspillen haben wir aufgezeigt.

Es bleibt unseres Erachtens falsch, Asarum mit den Aristolochia-Arten gleichzusetzen, denn es wirkt ganz anders und hat ganz andere Indikationen. Ausserdem enthält es viel weniger Aristolochiasäure und ist weder in Belgien noch in Taiwan in die Urothelkarzinom-Fälle involviert. Wir kommen deshalb zu dem Schluss, dass Asarum bei gewissen Erkältungen und Formen chronischer Kälte ohne Schaden verwendet werden kann. Asarum ist ein wichtiges Mittel, denn Erkältungen und Kälte sind in der Chinesischen Medizin keine Bagatelle, sondern können der Ausgangspunkt für schwere chronische Krankheiten sein.
(๑) 2013 S. Karger GmbH, Freiburg
Dr. med. Johannes G. Schmidt

Stiftung Paracelsus heute

Praxiszentrum Meinradsberg

Ilgenweidstrasse 3, 8840 Einsiedeln, Schweiz

schmidt@paracelsus-heute.ch 